EPJ Web of Conferences 41, 05020 (2013)

DOI: $10.1051 /$ epjconf/20134105020

(C) Owned by the authors, published by EDP Sciences, 2013

\title{
Ultrafast Pump-Push Photocurrent Spectroscopy of Organic Photoconversion Systems
}

\author{
A. A. Bakulin ${ }^{1,4}$, A. Rao ${ }^{1}$, Y. Vaynzof ${ }^{1}$, S. Gelinas ${ }^{1}$, V. G. Pavelyev ${ }^{2}$, P. H. M. van Loosdrecht ${ }^{2}$, M. \\ S. Pshenichnikov ${ }^{2}$, D. Niedzialek ${ }^{3}$, J. Cornil ${ }^{3}$, D. Beljonne ${ }^{3}$, and R. H. Friend ${ }^{1}$ \\ ${ }^{1}$ Cavendish laboratory, University of Cambridge, JJ Thomson Avenue, Cambridge CB30HE, UK \\ ${ }^{2}$ Zernike Institute for Advanced Materials, University of Groningen, Nijenborgh 4, 9747 AG \\ Groningen, The Netherlands \\ ${ }^{3}$ Laboratory for Chemistry of Novel Materials, University of Mons, Place du Parc 20, B-7000 Mons, \\ Belgium \\ ${ }^{4}$ AMOLF, Science Park 104, 1098 XG Amsterdam, The Netherlands
}

\begin{abstract}
Novel optical pump-push - photocurrent probe ultrafast spectroscopy experiments on organic photoconversion systems show that excessive excitation energy in such systems is not lost but used to reach delocalised states that act as the gateway for long-range charge separation. We also show that the developed experimental approach can be generalised to inorganic and hybrid photoconversion systems.
\end{abstract}

In organic light-to-charge conversion systems, photon absorption leads to the formation of a tightly bound electron-hole pair (Fig. 1a). In photosynthesis, separation of charges is achieved with the aid of cascaded energy levels and ion screening. Organic photovoltaic (OPV) devices in contrast possess none of these elements, yet surprisingly, some OPV systems work very efficiently. Hence, the fundamental mechanism of how the organic heterojunction enables efficient long-range charge separation remains unclear.

Here we address this issue by applying novel electro-optical pump-push-probe technique, performed on working OPV diodes (Fig. 1a,b) [1]. An ultrafast visible 'pump' pulse illuminates the OPV cell and creates a population of charged states. The charge carrier causes a geometrical rearrangement of organic molecules, known as polaron formation. For the hole polaron, the optical transition $\mathrm{P} 1$ between it and the $\pi$ valence band is strongly allowed, forming an absorption band typically between 0.2 and $0.6 \mathrm{eV}$ [2]. Here we selectively excited the P1 transition with an IR 'push' pulse, therefore donating excess energy to the system in different moments of photoconversion process. The perturbation of the charge dynamics on the molecular level affected the OPV device performance and the corresponding change of the photocurrent $(\delta \mathrm{PC})$ induced by the IR-'push' pulse is detected as a function of the delay between 'pump' and push [3]. In a complementary all-optical experiment, the effect of the 'push' pulse on charge dynamics is observed using an additional IR 'probe' pulse [4].

Figure 1c shows pump-push photocurrent data for a set of organic solar cells indicating a change in photocurrent when the push pulse arrives after the pump. The relative amount of additional photocurrent is very dependent on the material. Polymer-polymer blends (PFB/F8BT and P3HT/F8TBT) displayed a large push-induced increase of photocurrent (10-50\%). In contrast, highly efficient polymer-fullerene cells demonstrated a modest photocurrent increase $(<6 \%)$. The

This is an Open Access article distributed under the terms of the Creative Commons Attribution License 2.0, which permits unrestricted use, distribution, and reproduction in any medium, provided the original work is properly cited. 
magnitude of the signal decays slowly $(0.2-10 \mathrm{~ns})$ as the delay time increases, which we attribute to the bound-state recombination dynamics.
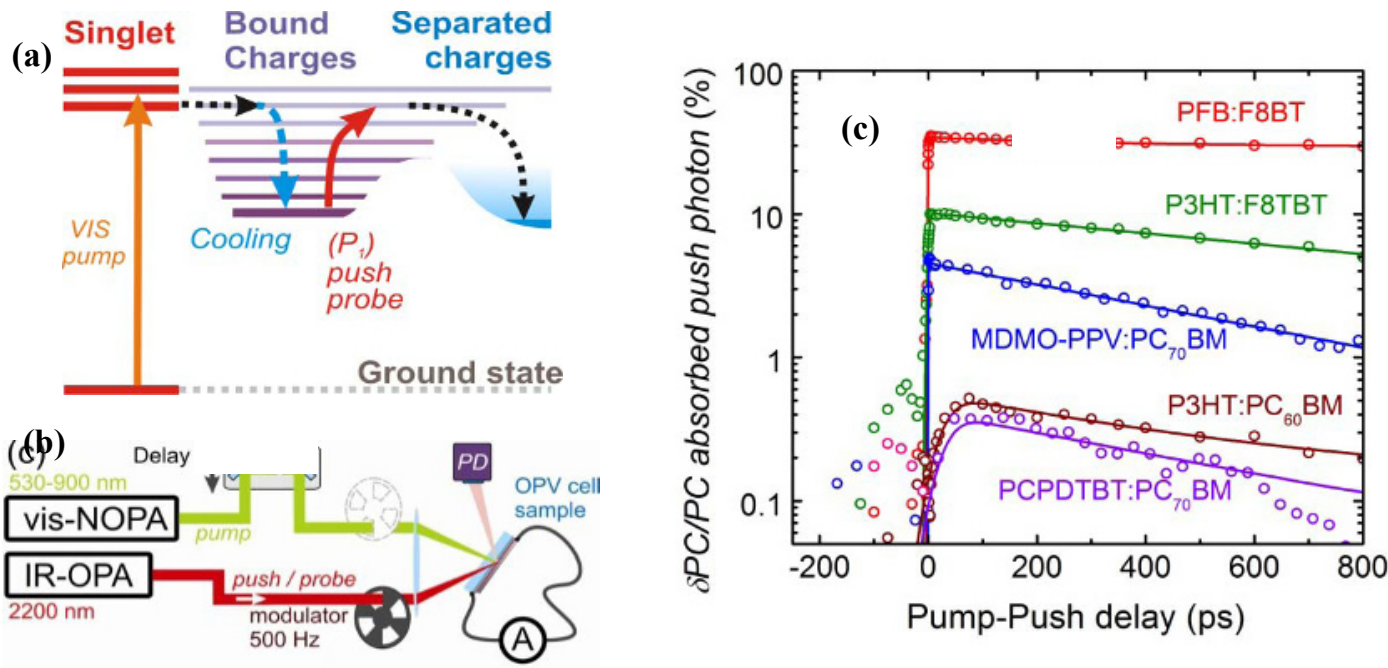

Fig.1. Free-energy diagram of photoconversion in organic systems (a) and layout of pump-push photocurrent (b) experiment. (c) Increase in photocurrent due to interaction with an IR push pulse for a set of OPV cells. Solid lines are exponential fits convoluted with the Gaussian 'charge-generation' function.

We monitor the effect of the 'push' pulse on the charges directly by an all-optical pumppush-probe technique (Fig. 1b). Pump pulses create charges in the OPV material and their evolution is manipulated /monitored with the IR 'push'/'probe' pulse.
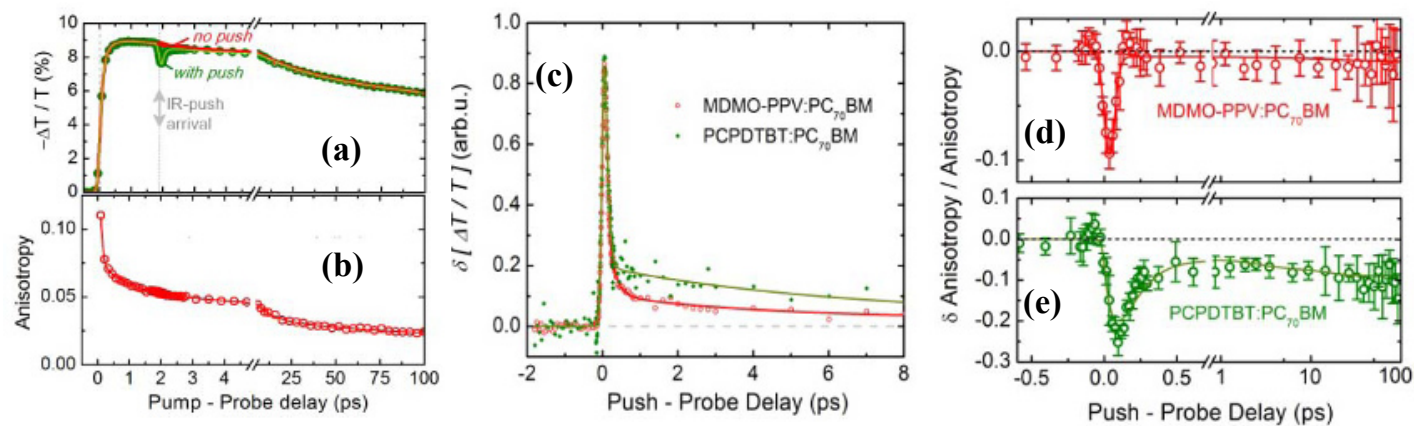

Fig. 2. (a) Transient-absorption and (b) transient-anisotropy kinetics for MDMO-PPV:PC ${ }_{70} \mathrm{BM}$ film excited at $580 \mathrm{~nm}$ and probed $3 \mu \mathrm{m}$. Green line in figure (a) shows dynamics with push pulse arriving at $\sim 2$ ps delay. The effect of push pulse on the isotropic (c) and anisotropic (d,e) transients, calculated from the difference between 'with-push' and 'no-push' measurements, for MDMO-PPV/ $\mathrm{PC}_{70} \mathrm{BM}$, $\mathrm{PCPDTBT} / \mathrm{PC}_{70} \mathrm{BM}$ films. In all figures, solid lines are (multi)exponential fits convoluted with the Gaussian function at zero delay.

Figures 2a,b show isotropic and anisotropy pump-probe transients for a typical OPV sample. The green circles present analogous dynamics when a push pulse, $\sim 2 \mathrm{ps}$ after the 'pump' deposits a $0.5 \mathrm{eV}$ excess energy to the charges, bringing them to the 'hot' state. This is observed as a sudden drop of signal amplitude because fewer charges absorb the probe beam. Figure 2c presents the difference in response with and without the 'push' pulse showing that deposited excess energy is lost on sub-ps time scale. Figures 2 d,e show the relative difference 
between 'with-push' and 'no-push' anisotropic transients. As a general trend, we observe that anisotropy is reduced by the 'push'. The loss in anisotropy after the push corresponds to the re-excited 'hot' charge re-localisation to the neighbouring molecule with a different orientation of the charge-associated dipole. The continued loss of anisotropy provides further evidences that charge carriers become more mobile after the 'push' pulse.
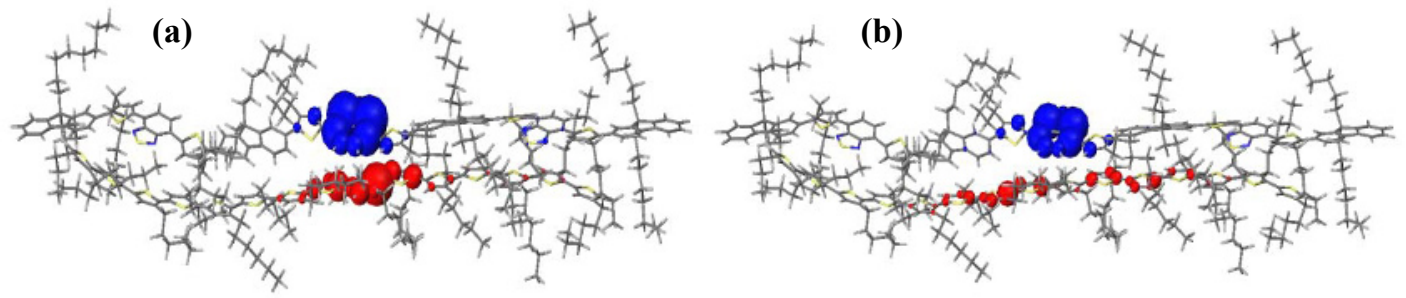

Fig. 3. Microelectrostatic simulations of the charge distribution at the P3HT/F8TBT heterojunction, with electron and hole densities shown in blue and red respectively. (a) the charge distribution in the lowest charged state configuration, and (b) the excited charged state configuration, created upon absorption of an IR-'push' photon.

The fast relaxation and anisotropy loss observed in 3-pulse experiments cannot be described by the currently dominant models of charge separation through relaxation-assisted intermolecular hopping. However, the observed results can be explained by charge delocalisation achieved via band states. The quantum chemistry calculations show that the absorption of the IR 'push' photon promotes charges from the localised polaronic state (Fig. $3 a)$ to the de-localised band state (Fig. 3b), increasing the effective radius of the charge pair and, therefore, reducing the binding energy.

In conclusion, by applying new types of ultrafast spectroscopes to range of organic photoconversion systems, we have demonstrated that delocalised band states are critical for long-range charge separation in OPV devices. Although the delocalised states are extremely short-lived $(<1 \mathrm{ps})$, they enable charges to override the otherwise dominant Coulomb interaction. Our results provide a new framework to understand charge generation in organic systems and outline the basis for the design of improved OPVs. In particular, we have already observed similar effects in different organic-base systems including hybrid and dye-sensitised light converters.[5]

\section{References}

1. A. A. Bakulin, A. Rao, V. G. Pavelyev, P. H. M. v. Loosdrecht, M. S. Pshenichnikov, D. Niedzialek, J. Cornil, D. Beljonne and R. H. Friend, Science 335, 1340 (2012)

2. J. Holt, S. Singh, T. Drori, Y. Zheng and Z. V. Vardeny, Physical Review B 79 (19) (2009)

3. J. G. Müller, J. M. Lupton, J. Feldmann, U. Lemmer, M. C. Scharber, N. S. Sariciftci, C. J. Brabec and U. Scherf, Phys. Rev. B 72, 195208 (2005)

4. K. C. Vishnubhatla, J. Clark, G. Lanzani, R. Ramponi, R. Osellame and T. Virgili, Applied Physics Letters 94 (4), 041123 (2009)

5. Y. Vaynzof, A.A. Bakulin, S. Gelinas, and R.H. Friend, Phys. Rev. Lett. 108, 246605 (2012) 